\title{
Status report on TAUOLA, its environment, and its applications
}

\author{
Z. Wạs ${ }^{a * \dagger}$, \\ ${ }^{a}$ Institute of Nuclear Physics, Radzikowsiego 152, 31-342 Cracow, Poland. \\ and \\ CERN, Theory Division, CH-1211 Geneva 23, Switzerland.
}

\begin{abstract}
The status of the Monte Carlo programs for the simulation of the $\tau$-lepton production and decay in high energy accelerator experiments is reviewed. In particular, the status of the following packages is discussed: (i) TAUOLA for $\tau$-lepton decay and PHOTOS for radiative corrections in decays, (ii) MC-TESTER for universal tests of the Monte Carlo programs describing particle decays, (iii) KORALB, KORALZ, KKMC packages for $\tau$-pair production in $e^{+} e^{-}$ collisions, and (iv) universal interface of TAUOLA for the decay of $\tau$-leptons produced by "any" generator.
\end{abstract}

CERN-TH/2002-302

\section{INTRODUCTION}

The package TAUOLA [1, 2, 3, , , th for the simulation of $\tau$-lepton decays and PHOTOS [5.60 for the simulation of radiative corrections in decays, have a rather long history. Written and maintained by well defined authors, they nonetheless migrated into a wide range of applications, where they became ingredients of complicated simulation chains. As a consequence, a large number of different versions are currently in use. From the algorithmic point of view, they often differ only in a few small details, but incorporate substantial amounts of specific results from distinct $\tau$-lepton measurements. Such versions were mainly maintained by the experiments taking precision data on $\tau$-leptons. On the other hand, many new applications were developed recently, often requiring program different versions because of interfaces to other packages.

In the following, I will concentrate on those topics where changes with respect to the status presented at the Victoria $\tau$ conference two years ago [7] were introduced. Since that time, there were no changes introduced into the PHOTOS Monte

\footnotetext{
*This work is partly supported by the Polish State Committee for Scientific Research (KBN) grants 2 P03B 001 22, and also by the European Community's Human Potential Programme under contract HPRN-CT-2000-00149 Physics at Colliders.

${ }^{\dagger}$ Home page at http://wasm.home.cern.ch/wasm/
}

Carlo functionality, and also the TAUOLA interfaces to KORALB [8,9], KORALZ [10], and KKMC [11] remain unchanged. On the contrary, the universal interface of TAUOLA evolved, and new applications, in particular observables for the measurement of the Higgs-boson parity, became possible. The new program MC-TESTER 12] instrumental in the development of future versions of TAUOLA, was developed. A new choice of hadronic currents for $\tau \rightarrow 4 \pi \nu$ decay modes became available. It is based on Novosibirsk data.

Let me concentrate, in the following three sections, on these topics, and close my contribution with a summary.

\section{THE $\tau$-LEPTON FOR HIGGS BOSON PARITY}

In many applications, the precision of the control of the $\tau$ spin effects is not crucial. This is the case in searches of new particles yet to be discovered, or in applications where $\tau$-lepton decays contribute as a final state of some rare decays of known particles. Nonetheless, in such cases, spin effects can be of some use as well. As these, there is no motivation to develop sophisticated spin algorithms for every individual case: less precise, but universal solutions are welcomed.

The universal solution of [13], based on the HEPEVT common block of FORTRAN77 is now dis- 
tributed with TAUOLA. The basic idea was to calculate the spin (helicity) state of the decaying $\tau$ from the kinematical information available in HEPEVT and some very simple assumptions on the production mechanisms. The program was checking if the production was through $f \bar{f} \rightarrow Z / \gamma \rightarrow \tau^{+} \tau^{-}, W$ or Higgs-boson intermediate states/processes. The main properties of the algorithm were presented in Victoria already 2 years ago; modifications necessary for the program to work in the case of some $\tau$ production processes involving new, to-be-discovered particles, have gradually been introduced, but one can see 114 that not in all cases is the effort completed.

In the meantime algorithm functionality was also extended: full spin effects were introduced in the case of Higgs-boson decay [15]. The case of the Higgs boson is exceptionally easy, since the full density matrix of the $\tau$-lepton pair produced from a Higgs-boson is fully defined by boson parity and $\tau$ leptons four-momenta.

This algorithm helped us to design an observable 16] for the possible measurement of Higgs boson parity in future accelerators such as Linear Colliders or the LHC. Let us recall the main principle of this technique, which from the point of view of spin analysis is quite involved, as it requires a study of full spin correlation spanning over three levels of decay cascade: $h / A \rightarrow \tau^{+} \tau^{-}$, $\tau^{ \pm} \rightarrow \rho^{ \pm} \nu$ and $\rho^{ \pm} \rightarrow \pi^{ \pm} \pi^{0}$. The presence of two non-observable neutrinos as well as our inability to reconstruct the Higgs-boson rest frame sufficiently well (with a precision comparable to the $\tau$-lepton mass) complicates the picture even further.

We started from the observation that the distribution of the acoplanarity angle of two planes spanned on decay products of $\rho^{+} \rightarrow \pi^{+} \pi^{0}$ and $\rho^{-} \rightarrow \pi^{-} \pi^{0}$ (defined in the rest frame of a $\rho^{+} \rho^{-}$ pair) is quite sensitive, see fig. 1, to the parity of Higgs boson. One has to select sub-samples of particles, with the same sign (opposite sign) of the energy difference between $\pi^{+}$and $\pi^{0}$ in the $\tau^{+}$rest frame and that between $\pi^{-}$and $\pi^{0}$ in the $\tau^{-}$rest frame. This condition is unfortunately impossible to realize in practice, as $\tau$-lepton momenta cannot be reconstructed.

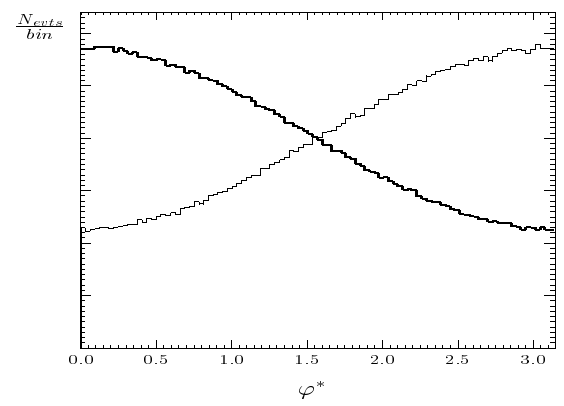

Figure 1. Acoplanarity distribution of the $\rho^{+} \rho^{-}$ decay products' in the rest frame of the $\rho^{+} \rho^{-}$pair. $A$ cut on the $\pi^{ \pm}$to $\pi^{0}$ energy difference defined, in the generator level $\tau^{ \pm}$rest frames, to be of the same sign is used. No smearings included. The thick line denotes the case of the scalar Higgsboson and the thin line the pseudoscalar one.

In the next step of our study we have weakened the requirement, and we have used a replacement for the $\tau$-lepton momentum; the $\rho^{+}$and $\rho^{-}$directions (in the rest frame of a $\rho^{+} \rho^{-}$pair) replace the unobservable directions of $\tau$ flights and the Higgs mass constraint provides the $\tau$-lepton energy (also in the same frame). Indeed, with such a technique, and after including some assumption on detector smearings, the sensitivity of our observable diminished significantly (see fig. 2), but nonetheless remained sizeable. Enough to

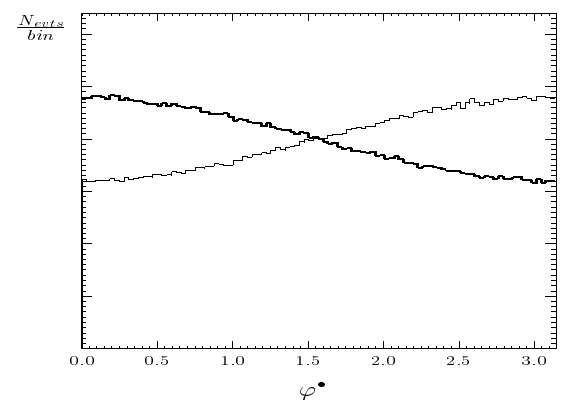

Figure 2. Acoplanarity distribution of the $\rho^{+} \rho^{-}$ decay products' in the rest frame of the $\rho^{+} \rho^{-}$pair. $A$ cut on the differences of the $\pi^{ \pm}$to $\pi^{0}$ energies defined, in their respective replacement $\tau^{ \pm}$rest frames, to be of the same sign is used. All detector smearings are included. The thick line denotes the case of the scalar Higgs boson and the thin line the pseudoscalar one. 
guarantee the meaurement of Higgs boson parity to a confidence level greater than $95 \%$, if typical assumptions on linear collider luminosity, and $(120 \mathrm{GeV})$ Higgs-boson production mechanism are made. Our method proved quite stable, with a degrading assumption on angular and energy resolution of the detector. Even a reduction by a factor of 2-3 with respect to typical assumptions used in linear collider designs did not destroy the method. To preserve its sensitivity, the angular resolution must be $\leq \mathcal{O}\left(m_{\rho} / E_{\rho}\right)$.

\section{MC-TESTER}

In the previous case the HEPEVT data structure was used to search for possible hard processes to calculate the spin state of the decaying $\tau$. One can search over an event record such as HEPEVT for other information as well. An essential step before attempting any sizeable rebuilding of the TAUOLA library is to automate at least some of its tests (such rebuilding may soon become necessary because of improving $\tau$-lepton data). Such a test-program may be useful for other applications, not necessarily related to generators of $\tau$-lepton decay, as well. That is why we have developed an individual program for that purpose. The idea behind MC-TESTER [12] is quite simple: the user loads an extra library and, after the generation of every event with his generator, calls MC-TESTER, specifying the identifier for the particle to be searched for over the whole event record and studied. A data file from such a run is formed.

In the second (analysis) step, data files from two runs of different Monte Carlo programs can be compared. The output is given in the form of a $\mathrm{LTT}_{\mathrm{E}} \mathrm{X}$ file, which includes a table of all decay modes found(see fig. 3). The table is not only a list of these modes, but also, of the branching ratios calculated from the two runs. Number quantifying maximum of shape differences is also given. This number is calculated as a maximum of the shape difference parameters (with a part of the code easy to identify) for all invariant mass distributions of the decay channel under study. We choose to take into consideration invariant mass of every subset of the decay products for
Found decay modes:

\begin{tabular}{|c|c|c|c|}
\hline \multirow[t]{2}{*}{ Decay channel } & \multicolumn{2}{|c|}{ Branching Ratio \pm Rough Errors } & \multirow{2}{*}{$\begin{array}{l}\text { Max. shape } \\
\text { dif. param. }\end{array}$} \\
\hline & Generator \#1 & Generator \#2 & \\
\hline$\tau^{-} \rightarrow \nu_{\tau} \pi^{0} \pi^{-}$ & $25.3683 \pm 0.0159 \%$ & $25.3085 \pm 0.0159 \%$ & 0.04375 \\
\hline$\tau^{-} \rightarrow e^{-\tilde{\nu_{e}} \nu_{\tau}}$ & $17.8479 \pm 0.0134 \%$ & $18.1093 \pm 0.0135 \%$ & 0.00000 \\
\hline$\tau^{-} \rightarrow \mu^{-} \widetilde{\nu_{\mu}} \nu_{\tau}$ & $17.3866 \pm 0.0132 \%$ & $17.6326 \pm 0.0133 \%$ & 0.00000 \\
\hline$\tau^{-} \rightarrow \nu_{\tau} \pi^{-}$ & $11.0768 \pm 0.0105 \%$ & $11.1765 \pm 0.0106 \%$ & 0.00000 \\
\hline$\tau^{-} \rightarrow \nu_{\tau} \pi^{0} \pi^{0} \pi^{-}$ & $9.1865 \pm 0.0096 \%$ & $9.1171 \pm 0.0095 \%$ & 0.09413 \\
\hline$\tau^{-} \rightarrow \nu_{\tau} \pi^{+} \pi^{-} \pi^{-}$ & $8.9837 \pm 0.0095 \%$ & $8.8828 \pm 0.0094 \%$ & 0.09368 \\
\hline$\overline{\tau^{-} \rightarrow \nu_{\tau} \pi^{0} \pi^{+} \pi^{-} \pi^{-}}$ & $4.2973 \pm 0.0066 \%$ & $4.5319 \pm 0.0067 \%$ & 0.30310 \\
\hline$\tau^{-} \rightarrow \nu_{\tau} \pi^{0} \pi^{0} \pi^{0} \pi^{-}$ & $1.0765 \pm 0.0033 \%$ & $1.0090 \pm 0.0032 \%$ & 0.00724 \\
\hline$\tau^{-} \rightarrow \nu_{\tau} K^{-}$ & $0.7202 \pm 0.0027 \%$ & $0.7138 \pm 0.0027 \%$ & 0.00000 \\
\hline$\tau^{-} \rightarrow \nu_{\tau} \pi^{0} \pi^{0} \pi^{+} \pi^{-} \pi^{-}$ & $0.4990 \pm 0.0022 \%$ & $0.0897 \pm 0.0009 \%$ & 0.00000 \\
\hline$\tau^{-} \rightarrow \nu_{\tau} \pi^{0} K^{-}$ & $0.4785 \pm 0.0022 \%$ & $0.4617 \pm 0.0021 \%$ & 0.00000 \\
\hline$\tau^{-} \rightarrow \nu_{\tau} K_{L}^{0} \pi^{-}$ & $0.4624 \pm 0.0022 \%$ & $0.4444 \pm 0.0021 \%$ & 0.00000 \\
\hline$\tau^{-} \rightarrow \nu_{\tau} \pi^{-} K_{S}^{0}$ & $0.4610 \pm 0.0021 \%$ & $0.4449 \pm 0.0021 \%$ & 0.00000 \\
\hline$\tau^{-} \rightarrow \nu_{\tau} \pi^{+} \pi^{-} K^{-}$ & $0.3902 \pm 0.0020 \%$ & $0.5051 \pm 0.0022 \%$ & 0.52330 \\
\hline$\tau^{-} \rightarrow \nu_{\tau} \pi^{0} \pi^{-} \eta$ & $0.1707 \pm 0.0013 \%$ & $0.1696 \pm 0.0013 \%$ & 0.00000 \\
\hline$\tau^{-} \rightarrow \nu_{\tau} \pi^{-} K^{+} K^{-}$ & $0.1704 \pm 0.0013 \%$ & $0.1509 \pm 0.0012 \%$ & 0.07360 \\
\hline$\tau^{-} \rightarrow \nu_{\tau} \pi^{0} K_{L}^{0} \pi^{-}$ & $0.1605 \pm 0.0013 \%$ & $0.2745 \pm 0.0017 \%$ & 0.92850 \\
\hline$\tau^{-} \rightarrow \nu_{\tau} \pi^{0} \pi^{-} K_{S}^{0}$ & $0.1592 \pm 0.0013 \%$ & $0.2734 \pm 0.0017 \%$ & 0.93657 \\
\hline$\tau^{-} \rightarrow \nu_{\tau} \gamma \pi^{0} \pi^{-}$ & $0.1559 \pm 0.0012 \%$ & $0.1303 \pm 0.0011 \%$ & 0.00000 \\
\hline$\tau^{-} \rightarrow \nu_{\tau} K_{L}^{0} \pi^{-} K_{S}^{0}$ & $0.1510 \pm 0.0012 \%$ & $0.0763 \pm 0.0009 \%$ & 0.00270 \\
\hline$\tau^{-} \rightarrow \nu_{\tau} K_{L}^{0} K^{-}$ & $0.1289 \pm 0.0011 \%$ & $0.0508 \pm 0.0007 \%$ & 0.00000 \\
\hline$\tau^{-} \rightarrow \nu_{\tau} K_{S}^{0} K^{-}$ & $0.1287 \pm 0.0011 \%$ & $0.0507 \pm 0.0007 \%$ & 0.00000 \\
\hline$\tau^{-} \rightarrow \nu_{\tau} \pi^{0} \pi^{0} \pi^{0} \pi^{+} \pi^{-} \pi^{-}$ & $0.1094 \pm 0.0010 \%$ & $0.0506 \pm 0.0007 \%$ & 0.00000 \\
\hline$\tau^{-} \rightarrow \nu_{\tau} \pi^{+} \pi^{+} \pi^{-} \pi^{-} \pi^{-}$ & $0.0803 \pm 0.0009 \%$ & $0.0401 \pm 0.0006 \%$ & 0.00000 \\
\hline$\tau^{-} \rightarrow \nu_{\tau} \pi^{0} \pi^{0} K^{-}$ & $0.0792 \pm 0.0009 \%$ & $0.0504 \pm 0.0007 \%$ & 0.29190 \\
\hline$\tau^{-} \rightarrow \nu_{\tau} K_{L}^{0} K_{L}^{0} \pi^{-}$ & $0.0760 \pm 0.0009 \%$ & $0.0372 \pm 0.0006 \%$ & 0.00854 \\
\hline$\tau^{-} \rightarrow \nu_{\tau} \pi^{-} K_{S}^{0} K_{S}^{0}$ & $0.0756 \pm 0.0009 \%$ & $0.0378 \pm 0.0006 \%$ & 0.01189 \\
\hline$\tau^{-} \rightarrow \nu_{\tau} \pi^{0} K_{L}^{0} K^{-}$ & $0.0507 \pm 0.0007 \%$ & $0.0763 \pm 0.0009 \%$ & 0.85321 \\
\hline$\tau^{-} \rightarrow \nu_{\tau} \pi^{0} K_{S}^{0} K^{-}$ & $0.0498 \pm 0.0007 \%$ & $0.0746 \pm 0.0009 \%$ & 0.87506 \\
\hline$\tau^{-} \rightarrow \nu_{\tau} \pi^{0} \pi^{+} \pi^{+} \pi^{-} \pi^{-} \pi^{-}$ & $0.0186 \pm 0.0004 \%$ & $0.0293 \pm 0.0005 \%$ & 0.00000 \\
\hline
\end{tabular}

Figure 3. Second page of MC-TESTER booklet produced at analysis step.

the decay channel. The table is followed by a booklet of these invariant mass distributions (see fig. $⿴$ for an example of an individual plot), which are grouped into separate chapters for every decay mode.

\section{TAUOLA}

As was already discussed in Victoria [7], many options of physics initialization are now available for TAUOLA (they can be constructed from the files included in the program distribution package). They often differ by the models used for fits to the experimental data, and/or by the data themselves. Let me stress that, in general, the best 


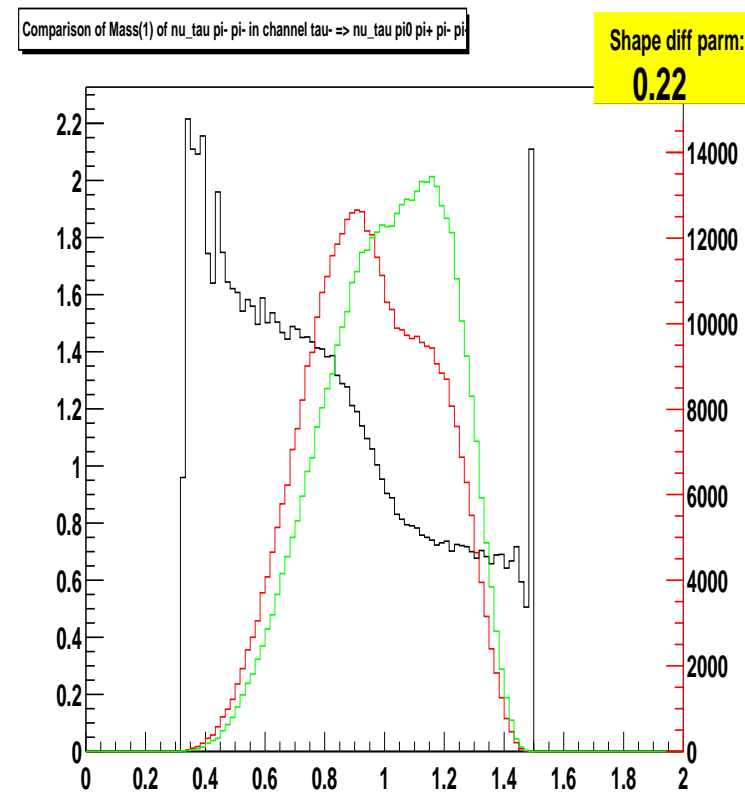

Figure 4. Example of individual plot from analysis step of MC-TESTER.

parametrizations will always be in the hands of the leading $\tau$-lepton experiments of the time. Our original version of TAUOLA initialization was indeed meant to be used only after re-initialization of its physics content by the users. Nonetheless for experiments in the design phase or for which $\tau$-leptons physical processes help in other studies, such a solution is not good. We are gradually including new options into TAUOLA.

In the last two years, parametrizations of the hadronic form factors [17] based on the Novosibirsk low energy $e^{+} e^{-}$annihilation data [18] were introduced into TAUOLA as a possible new option for $\tau^{-} \rightarrow \pi^{-} \pi^{-} \pi^{+} \pi^{0} \nu$ and $\tau^{-} \rightarrow \pi^{-} \pi^{0} \pi^{0} \pi^{0} \nu$ decay modes 19 . This is the only published contribution documenting upgrades on the physics content of TAUOLA over the last two years.

\subsection{Comment on one of the old tests}

In ref. [3] (formulae (37) and (38)) we have reported that, for $\tau$ decay into $4 \pi$, when the chiral limit is taken (the case we used for some tests of phase-space generation), the results of TAUOLA agree with those of ref. 20, only up to an overall factor $\frac{243}{2 \cdot n !} \cos ^{2} \theta_{c}$. In the case of

${ }^{3}$ Let us note that problems with normalization_or/and with misprints) in [20] were again addressed [21]. The $\tau \rightarrow \pi^{-} \pi^{-} \pi^{+} \pi^{0} \nu,(n=2)$ the test formula

$\frac{\Gamma\left(2 \pi^{-} \pi^{+} \pi^{0}\right)}{\Gamma_{e}}=\frac{\cos ^{2} \theta_{c}}{15}\left(\frac{m_{\tau}}{2 \pi f_{\pi}}\right)^{4} \frac{1}{128} C_{N}$

returns 0.0271364 with the numerical coefficient $C_{N}=1261 / 120-\pi^{2}$ (for compatibility, we use as in [3] $m_{\tau}=1.7842, f_{\pi}=0.0933$ and $\cos \theta_{c}=0.975$ ). A later calculation (formula 33 in [23]) finds $C_{N}=1009 / 96-\pi^{2}$ and result is 0.0272249 . With the computer power available at the time of [3], we could not distinguish between the two options. At present, such an exercise is straightforward and we find that TAUOLA returns $0.0272060 \pm 0.000011$. We can see, that the value of $C_{N}$ from ref. [23] is statistically favoured over the one from ref. 20.

\section{SUMMARY}

Let me recall finally the main developments of the TAUOLA package in the last two years: as stand-alone generator of $\tau$-lepton decays, and for its interfaces to other programs. The progress on $\tau$-lepton decay alone consists of a new parametrization of form factors for the $4 \pi$ decay channels. This parametrization, mainly based on the Novosibirsk data, form a step toward TAUOLA as a framework, where different data and models can be compared. In such multi parametrization approach the appropriate tools are necessary. The MC-TESTER, helpful in different types of works for programs such as TAUOLA became available. It can also be useful, for example, to check if the interfacing of TAUOLA to other programs did not corrupt its initialization. The family of TAUOLA interfaces was enriched with the new option for its universal interface. It now provides complete spin correlations for Higgs boson decay into pair of $\tau$ leptons. This option was instrumental in

authors of that paper worry that it may be of grave consequences for TAUOLA as well. In practice, even if the normalization of the chiral current was wrong (by the same factor in the Monte Carlo and in the testing function), it would affect neither technical tests nor actual results of TAUOLA generation, since a different current is used then. Note that in cases such as CLEO initialization [22], the current is normalized directly from the $\tau$ data collected by the experiments; the normalization originating from the model disappears completely. This is why the issue is only of marginal interest for TAUOLA as it is now. 
designing an observable that is potentially useful to measure Higgs-boson parity at future experiments.

Let me finally acknowledge people and collaborations who contributed to the present shape of TAUOLA and related projects: A. Bondar, S. Eidelman, P. Golonka, S. Jadach, M. Jeżabek, J.H. Kühn, A. Milstein, T. Pierzchala, E. RichterWas, N. Root, B.F.L. Ward, M. Worek, and ALEPH, CLEO (in particular A. Weinstein), Delphi, Opal, L3 collaborations, the Karlsruhe theory group, and others. I would like to thank A. Pich for discussion.

\section{REFERENCES}

1. S. Jadach, J. H. Kühn and Z. Wąs, Comput. Phys. Commun. 64 (1990) 275.

2. M. Jeżabek, Z. Wąs, S. Jadach and J. H. Kühn, Comput. Phys. Commun. 70 (1992) 69.

3. S. Jadach, Z. Wạs, R. Decker and J. H. Kühn, Comput. Phys. Commun. 76 (1993) 361-380.

4. P. Golonka, E. Richter-Wạs and Z. Wạs, hep-ph/0009302.

5. E. Barberio, B. van Eijk and Z. Wạs, Comput. Phys. Commun. 66 (1991) 115.

6. E. Barberio and Z. Wąs, Comput. Phys. Commun. 79 (1994) 291-308.

7. Z. Wạs, Nucl. Phys. Proc. Suppl. 98 (2001) 96-102, hep-ph/0011305.

8. S. Jadach and Z. Wąs, Comput. Phys. Commun. 85 (1995) 453-462.

9. S. Jadach and Z. Wạs, Acta Phys. Polon. B15 (1984) 1151; Erratum: B16 (1985) 483.

10. S. Jadach, B. F. L. Ward and Z. Wa̧s, Comput. Phys. Commun. 79 (1994) 503.

11. S. Jadach, B. F. L. Ward and Z. Wąs, Comput. Phys. Commun. 130 (2000) 260; an up-to-date source is available from http://home.cern.ch/jadach/.

12. P. Golonka, T. Pierzchała and Z. Wạs, hep-ph/0210252.

13. T. Pierzchała, E. Richter-Wa̧s, Z. Wa̧s and M. Worek, Acta Phys. Polon. B32 (2001) 1277-1296, hep-ph/0101311.

14. C. Biscarat, web page at http: //home.cern.ch/cbiscara/tauola_valid.html.
15. Z. Wạs and M. Worek, Acta Phys. Polon. B33 (2002) 1875-1884, hep-ph/0202007.

16. G. R. Bower, T. Pierzchała, Z. Wa̧s and M. Worek, Phys. Lett. B543 (2002) 227-234, hep-ph/0204292.

17. A. E. Bondar, S. I. Eidelman, A. I. Milstein and N. I. Root, Phys. Lett. B466 (1999) 403407, hep-ph/9907283.

18. CMD2 Collaboration, R. R. Akhmetshin et al., Phys. Lett. B466 (1999) 392-402, hep-ex/9904024.

19. A. E. Bondar et al., Comput. Phys. Commun. 146 (2002) 139-153, hep-ph/0201149.

20. R. Fischer, J. Wess and F. Wagner, Z. Phys. C3 (1980) 313-320.

21. G. Ecker and R. Unterdorfer, hep$\mathrm{ph} / 0209056$.

22. CLEO Collaboration, A. Weinstein, see http: //www.cithep.caltech.edu/ ajw/korb_doc. html\#files.

23. H. Czyż and J. H. Kühn, Eur. Phys. J. C18 (2001) 497-509, hep-ph/0008262. 